\title{
A Critical Look at Microprocessor Architecture
}

\author{
Organizer/Moderator: Carver A. Mead, Cal. Inst. of Technology, Pasadena, CA
}

\begin{abstract}
LSI has provided machine designers a medium of unprecedented power and versatility. The potential of this technology has been doubling every year and promises to continue for another factor of 1000 . It is inevitable that such potential will be used to achieve ever more powerful machine organizations and will, in the long run, totally change our basic concepts of machine structure and function. Todate, however, the microprocessors which have been implemented are of a very conventional type. This trend poses several fundamental questions.
\end{abstract}

(1)- Will this remarkable opportunity for innovation be submerged in the clamor for standardization?

(2)- Can (and will) machine organizations allow incremental addition of functional complexity without necessitating a software (and hardware) start from scratch at each point?

(3)- When (and how) will peripherals come to be viewed as a central rather than peripheral, part of machine design and programming?

(4)- Should all machine functions (such as memory address maintenance, conditional branching, peripheral control, etc.) flow through a CPU designed primarily for arithmetic and logical operations on data?

(5)- What are the advantages (and disadvantages) in separating data memary from program memory?

(6). In what environments should a high level instruction set be part of the machine hardware?

These and related questions will form the basis for discussion focussed on the definition, design, and use of these remarkable machines of the future.

\section{Panel Members}

\author{
T. Bennett, Motorola Semiconductor Products, Phoenix, AZ \\ F. Faggin, Intel Corp., Santa Clara, CA
}

W. Roberts, Western Digital Corp., Newport Beach, CA

J. Ogdin, Microputer Techniques, Inc., Reston, VA 